\title{
ON THE EXISTENCE OF SOLUTIONS OF STRONGLY DAMPED NONLINEAR WAVE EQUATIONS
}

\author{
JONG YEOUL PARK and JEONG JA BAE
}

(Received 18 June 1998)

$$
\begin{aligned}
& \text { ABSTRACT. We investigate the existence and uniqueness of solutions of the following equa- } \\
& \text { tion of hyperbolic type with a strong dissipation: } \\
& \qquad \begin{array}{r}
u_{t t}(t, x)-\left(\alpha+\beta\left(\int_{\Omega}|\nabla u(t, y)|^{2} d y\right)^{\gamma}\right) \Delta u(t, x) \\
\quad-\lambda \Delta u_{t}(t, x)+\mu|u(t, x)|^{q-1} u(t, x)=0, \quad x \in \Omega, t \geq 0, \\
u(0, x)=u_{0}(x), \quad u_{t}(0, x)=u_{1}(x), \quad x \in \Omega,\left.u\right|_{\partial \Omega}=0,
\end{array}
\end{aligned}
$$

where $q>1, \lambda>0, \mu \in \mathbb{R}, \alpha, \beta \geq 0, \alpha+\beta>0$, and $\Delta$ is the Laplacian in $\mathbb{R}^{N}$.

Keywords and phrases. Quasilinear wave equation, nondegenerate equation, existence and uniqueness, continuously differentiable solution, variation of parameters representation.

2000 Mathematics Subject Classification. Primary 35L70, 35L15, 65M60.

1. Introduction. Let $\Omega$ be a bounded domain in $\mathbb{R}^{N}$ with smooth boundary $\partial \Omega$. In this paper, we consider the initial boundary value problem for the second order equations:

$$
\begin{aligned}
u_{t t}(t, x)-\left(\alpha+\beta\left(\int_{\Omega}|\nabla u(t, y)|^{2} d y\right)^{\gamma}\right) \Delta u(t, x) & \\
-\lambda \Delta u_{t}(t, x)+\mu|u(t, x)|^{q-1} u(t, x)=0, & x \in \Omega, t \geq 0, \\
u(0, x) & =u_{0}(x), \quad u_{t}(0, x)=u_{1}(x), \quad x \in \Omega,\left.\quad u\right|_{\partial \Omega}=0,
\end{aligned}
$$

where $q>1, \lambda>0, \mu \in \mathbb{R}, \alpha, \beta \geq 0, \alpha+\beta>0$, and $\Delta$ is the Laplacian in $\mathbb{R}^{N}$.

Equation (1.1) has its origin in the nonlinear vibrations of an elastic string (cf. Narasimha [6]). We call equation (1.1) a nondegenerate equation when $\alpha>0$ and $\beta>0$ and a degenerate one when $\alpha=0$ and $\beta>0$. In the case of $\alpha>0$ and $\beta=0$, equation (1.1) is the usual semilinear wave equations.

Many authors have studied the existence and uniqueness of solutions of (1.1) by using various methods. When $\lambda>0$ and $\mu=0$, for the degenerate case (i.e., $\alpha=0$ ), Nishihara and Yamada [7] have proved the global existence of a unique solution under the assumptions that the initial data $\left\{u_{0}, u_{1}\right\}$ are sufficiently small and $u_{o} \neq 0$. However, the method in [7] cannot be applied directly to the case that degenerate equations have the blow-up term $|u|^{q-1} u$. When $\alpha>0$ and $\mu>0$, for the degenerate case (i.e., $\alpha=0$ ), Ono and Nishihara [9] have proved the global existence and decay 
structure of solutions of (1.1) without small condition of initial data using Galerkin method. Ono [8] has obtained the global existence of solutions of problem (1.1) with dissipative term $u^{\prime}$ instead of $\Delta u^{\prime}$. In this paper, we prove the existence of solutions of problem (1.1) using the method of Fitzgibbon and Parrot [3].

Our plan in this paper is as follows: in Section 2, we collect the results about abstract semigroup theory and present some lemmas. In Section 3, we deal with a priori estimates for solutions of (1.1) and in Section 4, we investigate convergence of solution.

2. Preliminaries. In this section, we formulate (1.1) as abstract Cauchy initial value problems. We denote by $H$ the Hilbert space $L^{2}(\Omega)$ with norm $\|\cdot\|_{2}$ and inner product $(\cdot, \cdot)$. We define $A: D(A) \subset H \rightarrow H$ by

$$
A u=-\Delta u \text { for } u \in D(A),
$$

where

$$
D(A)=H^{2}(\Omega) \cap H_{0}^{1}(\Omega) .
$$

Here, $H_{0}^{1}(\Omega)$ and $H^{2}(\Omega)$ are the usual Sobolev spaces. It is well known that $A$ so defined is a strictly positive selfadjoint operator on $H$. Positive powers of $A, A^{\gamma}$ for $\gamma>0$, may be computed via the elementary spectral calculus and are seen to be positive selfadjoint operators themselves. We can make $D\left(A^{\gamma}\right)$ into a Hilbert space $H_{A^{\gamma}}$ by imposing a graph norm

$$
\|u\|_{A^{\gamma}}=\left\|A^{\gamma} u\right\|_{2} \quad \text { for } u \in D\left(A^{\gamma}\right) .
$$

It should be evident that the damped beam equation (1.1) may be written abstractly as

$$
\begin{gathered}
u^{\prime \prime}(t)+\left(\alpha+\beta\left\|A^{1 / 2} u(t)\right\|_{2}^{2 \gamma}\right) A u(t)+\lambda A u^{\prime}(t)+\mu|u(t)|^{q-1} u(t)=0, \quad t \geq 0, \\
u(0)=u_{0}, \quad u^{\prime}(0)=u_{1},\left.\quad u\right|_{\partial \Omega}=0 .
\end{gathered}
$$

In order to prove the existence of solutions of (1.1), first we consider the following initial value problem:

$$
\begin{gathered}
u^{\prime \prime}(t)+\left(\alpha+\beta\left\|A^{1 / 2} u(t)\right\|_{2}^{2 \gamma}\right) A u(t)+\epsilon A^{2} u(t)+\lambda A u^{\prime}(t)+\mu|u(t)|^{q-1} u(t)=0, \quad t \geq 0, \\
u(0)=u_{0}, \quad u^{\prime}(0)=u_{1},\left.\quad u\right|_{\partial \Omega}=0 .
\end{gathered}
$$

Now, it is convenient to resort to the standard artifice of writing (2.5) as a first order system. We let $X=H_{A} \times H$ and define $\bar{A}_{\epsilon} ; X \rightarrow X$ by the operator matrix

$$
\bar{A}_{\epsilon}=\left(\begin{array}{cc}
0 & -I \\
\epsilon A^{2} & \lambda A
\end{array}\right) \quad \text { with } D\left(\bar{A}_{\epsilon}\right)=D\left(A^{2}\right) \times D(A) \equiv D_{1} .
$$

Proposition 2.1 [3]. If $\bar{A}_{\epsilon}$ is defined by (2.6), then $-\bar{A}_{\epsilon}$ is the infinitesimal generator of an analytic semigroup $\left\{\bar{T}_{\epsilon}(t)\right\} \in X,\left\{\bar{T}_{\epsilon}(t)\right\}$ is an analytic semigroup of contractions in $X_{\epsilon}=H_{\sqrt{\epsilon} A} \times H$. 
We note that while $\left\{\bar{T}_{\epsilon}(t) \mid t \geq 0\right\}$ is an analytic semigroup in $X$, we can no longer claim that it is a contraction semigroup. In fact, due to the singularity imposed by the factor $\epsilon$, we expect the norm of $\bar{T}_{\epsilon}(t)$ in $X$ to blow up as $\epsilon \rightarrow 0$. Now we define a nonlinear operator

$$
F_{0}\left(U_{\epsilon}\right)=\left(\begin{array}{c}
0 \\
-\left(\alpha+\beta\left\|A^{1 / 2} u\right\|^{2 \gamma}\right) A u-\mu|u|^{q-1} u
\end{array}\right) \text { for } U_{\epsilon}=\left(\begin{array}{l}
u \\
v
\end{array}\right) \in X .
$$

Then solutions to (2.5) now assume the form

$$
\begin{gathered}
\frac{d}{d t} U_{\epsilon}+\bar{A}_{\epsilon} U_{\epsilon}=F_{0}\left(U_{\epsilon}\right), \quad t>0, \\
U_{\epsilon}(0)=U_{0}=\left(u_{0}, u_{1}\right)^{T}
\end{gathered}
$$

We point out that if $\pi_{1}$ and $\pi_{2}$ project $X$ onto its first and second coordinates, respectively, then $\pi_{1} U_{\epsilon}(t)=u(t)$ and $\pi_{2} U_{\epsilon}(t)=u^{\prime}(t)$, where $u(t)$ is the strong solution of (2.5). We have the following results.

Proposition 2.2. If $U_{0}=\left(u_{0}, u_{1}\right)^{T} \in D_{1}$, then there exists a strong, continuously differentiable solution to $(2.8)$ on $[0, \infty)$ which has variation of parameters representation

$$
U_{\epsilon}(t)=\bar{T}_{\epsilon}(t) U_{0}+\int_{0}^{t} \bar{T}_{\epsilon}(t-s) F_{0}\left(U_{\epsilon}(s)\right) d s .
$$

Proof. Since the mapping $F_{0} ; X \rightarrow X$ is $C^{\infty}$, mild solution $u$ is Hölder continuous and so it is continuously differentiable (cf. Pazy [10, Chapter 4]).

We have pointed out that one expects the norm of $\bar{T}_{\epsilon}(t)$ in $X$ denoted by $\left\|\bar{T}_{\epsilon}\right\|_{X}$ to blow up as $\epsilon \rightarrow 0$ (although the norm in $X_{\epsilon},\left\|\bar{T}_{\epsilon}\right\|_{X_{\epsilon}}$ remains bounded by one ). For this reason, we want to prove an alternative representation to (2.9) for solutions to (2.8). We define

$$
\bar{A}=\left(\begin{array}{cc}
0 & -I \\
\alpha A & \lambda A
\end{array}\right) \quad \text { with } D(\bar{A})=D(A) \times D(A) .
$$

It is well known that $-\bar{A}$ is the infinitesimal generator of an analytic semigroup on $X$. (cf. Webb [12, Proposition 2.2]). We denote the semigroup generated by $-\bar{A}$ as $\{T(t) ; t \geq 0\}$ and introduce a new nonlinearity $F_{\epsilon}$ defined by

$$
F_{\epsilon}\left(U_{\epsilon}\right)=\left(\begin{array}{c}
0 \\
-\beta\left\|A^{1 / 2} u\right\|_{2}^{2 \gamma}-\epsilon A^{2} u-\mu|u|^{q-1} u
\end{array}\right) \text { for } U_{\epsilon}=\left(\begin{array}{c}
u \\
u^{\prime}
\end{array}\right) \in X .
$$

By merely regrouping terms, we can rewrite (2.8) as

$$
\begin{gathered}
\frac{d}{d t} U_{\epsilon}+\bar{A} U_{\epsilon}=F_{\epsilon}\left(U_{\epsilon}\right), \quad t>0, \\
U_{\epsilon}(0)=U_{0}=\left(u_{0}, u_{1}\right) .
\end{gathered}
$$

We have the following proposition. 
Proposition 2.3. If $U_{0}=\left(u_{0}, u_{1}\right)^{T} \in D_{1}$, then the strong solution to (2.12) may be represented as

$$
U_{\epsilon}(t)=T(t) U_{0}+\int_{0}^{t} T(t-s) F_{\epsilon}\left(U_{\epsilon}(s)\right) d s .
$$

Proof. We let $f_{\epsilon}(t)=F_{\epsilon}\left(U_{\epsilon}(t)\right)$ and refer to Pazy [10, page 106] for a discussion of the representation theory for the inhomogeneous Cauchy initial value problems of the form

$$
\frac{d}{d t} U_{\epsilon}+\bar{A}_{\epsilon} U_{\epsilon}=f_{\epsilon}(t), \quad t>0, \quad U_{\epsilon}(0)=U_{0}
$$

Now we turn our attention to (1.1). We know that there exists a unique strong solution $u(t, x)$ to $(1.1)$ if $\left(u_{0}, u_{1}\right) \in D\left(A^{1 / 2}\right) \times H$. Moreover, if $T>0$ and $(u(t))(x)=$ $u(t, x)$, then

$$
u \in L^{\infty}\left((0, T) ; H_{A^{1 / 2}}\right) \quad \text { and } \quad u^{\prime} \in L^{\infty}((0, T) ; H) \cap L^{2}\left((0, T) ; H_{A^{1 / 2}}\right) .
$$

See Ono [8] and Matos and Pereira [4].

The abstract second order equation

$$
\begin{gathered}
u^{\prime \prime}(t)+\alpha A u(t)+\beta\left\|A^{1 / 2} u(t)\right\|_{2}^{2 \gamma} A u(t)+\lambda A u^{\prime}(t)+\mu|u(t)|^{q-1} u(t)=0, \quad t \geq 0, \\
u(0)=u_{0}, \quad u^{\prime}(0)=u_{1}
\end{gathered}
$$

places (1.1) in a function space setting. We introduce a nonlinear operator $F$ defined by

$$
F(U)=\left(\begin{array}{c}
0 \\
-\beta\left\|A^{1 / 2} u\right\|_{2}^{2 \gamma} A u-\mu|u|^{q-1} u
\end{array}\right) \text { for } U=\left(\begin{array}{c}
u \\
u^{\prime}
\end{array}\right)
$$

and observe that solutions to (1.1) satisfy

$$
\frac{d}{d t} U+\bar{A} U(t)=F(U(t)), \quad t>0, \quad U(0)=U_{0}=\left(u_{0}, u_{1}\right)^{T} .
$$

We have the following proposition which we state without proof.

Proposition 2.4 [10]. If $U_{0}=\left(u_{0}, u_{1}\right)^{T} \in D_{1}$, then there exists a strong solution to (1.1) on $[0, \infty)$ which has abstract variation of parameters representation

$$
U(t)=T(t) U_{0}+\int_{0}^{t} T(t-s) F(U(s)) d s
$$

3. A priori estimates. We first prepare the following well-known lemmas which are needed later.

LEMmA 3.1 (Sobolev-Poincaré [5]). If either $1 \leq q<+\infty$ where $N=1,2$ or $1 \leq q \leq$ $(N+2) /(N-2)$ where $N \geq 3$, then there is a constant $C(\Omega, q+1)$ such that

$$
\|u\|_{q+1} \leq C(\Omega, q+1)\|\nabla u\|_{2} \quad \text { for } u \in H_{0}^{1}(\Omega) .
$$


In other words,

$$
C(\Omega, q+1)=\sup \left\{\frac{\|u\|_{q+1}}{\|\nabla u\|_{2}} \mid u \in H_{0}^{1}(\Omega), u \neq 0\right\}
$$

is positive and finite.

LEMMA 3.2 (Gagliardo-Nirenberg [5]). Let $1 \leq r<q \leq+\infty$ and $p \leq q$. Then the inequality

$$
\|u\|_{W^{k, q}} \leq C\|u\|_{W^{m, p}}^{\theta}\|u\|_{r}^{1-\theta} \quad \text { for } u \in W^{m, p}(\Omega) \cap L^{r}(\Omega)
$$

holds with some $C>0$ and

$$
\theta=\left(\frac{k}{N}+\frac{1}{r}-\frac{1}{q}\right)\left(\frac{m}{N}+\frac{1}{r}-\frac{1}{p}\right)^{-1}
$$

provided that $0<\theta \leq 1$ (we assume that $0<\theta<1$ if $q=+\infty$ ).

Now, we develop a priori estimates for solutions to (1.1) by applying energy methods to abstract second order equations. Throughout this section, let $u$ be the solution to (2.5).

Proposition 3.3. If $\left(u_{0}, u_{1}\right)^{T} \in D_{1}$, then there exists a positive constant $M_{0}$, which does not depend on $\epsilon$, so that

$$
\sup _{t>0}\left\{\left\|u^{\prime}(t)\right\|_{2}^{2},\left\|A^{1 / 2} u(t)\right\|_{2}^{2}, \epsilon\|A u(t)\|_{2}^{2}\right\} \leq M_{0} .
$$

Proof. If we multiply equation (2.5) by $u^{\prime}(t)$ and integrate in space, we obtain

$$
\begin{aligned}
\frac{1}{2} \frac{d}{d t}\left(\left\|u^{\prime}(t)\right\|_{2}^{2}\right. & \left.+\alpha\left\|A^{1 / 2} u(t)\right\|_{2}^{2}+\epsilon\|A u(t)\|_{2}^{2}+\frac{2 \mu}{q+1}\|u(t)\|_{q+1}^{q+1}\right) \\
& +\frac{\beta}{2(\gamma+1)} \frac{d}{d t}\left\|A^{1 / 2} u(t)\right\|_{2}^{2(\gamma+1)}+\lambda\left\|A^{1 / 2} u^{\prime}(t)\right\|_{2}^{2}=0 .
\end{aligned}
$$

We may integrate (3.6) with respect to $t>0$ to obtain the desired result (3.5).

We obtain greater regularity of solutions by placing additional smoothness requirements on our initial data.

Proposition 3.4. If $\left(u_{0}, u_{1}\right)^{T} \in D\left(A^{2}\right) \times D(A)$ and

$$
\frac{N}{N-2} \leq q \leq \min \left\{\frac{N+2}{N-2}, \frac{N-2}{[N-4]^{+}}\right\} \quad(N \geq 3),
$$

then there exists a positive constant $M_{1}$, which does not depend on $\epsilon$, so that

$$
\sup _{t>0}\left\{\left\|A^{1 / 2} u^{\prime}(t)\right\|_{2}^{2},\|A u(t)\|_{2}^{2}, \epsilon\left\|A^{3 / 2} u(t)\right\|_{2}^{2}\right\} \leq M_{1} .
$$

Proof. Multiplying equation (2.5) by $A u^{\prime}(t)$,

$$
\begin{aligned}
& \frac{1}{2} \frac{d}{d t}\left(\left\|A^{1 / 2} u^{\prime}(t)\right\|_{2}^{2}+\alpha\|A u(t)\|_{2}^{2}+\epsilon\left\|A^{3 / 2} u(t)\right\|_{2}^{2}\right)+\lambda\left\|A u^{\prime}(t)\right\|_{2}^{2} \\
& \quad+\mu\left(A^{1 / 2}\left[|u(t)|^{q-1} u(t)\right], A^{1 / 2} u^{\prime}(t)\right)+\frac{\beta}{2}\left\|A^{1 / 2} u(t)\right\|_{2}^{2 \gamma} \frac{d}{d t}\|A u(t)\|_{2}^{2}=0 .
\end{aligned}
$$


Integrating (3.9) from 0 to $t$, we get

$$
\begin{aligned}
\frac{1}{2}\left\|A^{1 / 2} u^{\prime}(t)\right\|_{2}^{2}+\frac{\alpha}{2}\|A u(t)\|_{2}^{2}+\frac{\beta}{2}\left\|A^{1 / 2} u(t)\right\|_{2}^{2 \gamma}\|A u(t)\|_{2}^{2}+\frac{\epsilon}{2}\left\|A^{3 / 2} u(t)\right\|_{2}^{2} \\
+\mu \int_{0}^{t}\left(A^{1 / 2}\left[\left|u^{\prime}(s)\right|^{q-1} u(s)\right], A^{1 / 2} u^{\prime}(s)\right) d s+\lambda \int_{0}^{t}\left\|A u^{\prime}(s)\right\|_{2}^{2} d s \\
=\frac{1}{2}\left\|A^{1 / 2} u_{1}\right\|_{2}^{2}+\frac{\alpha}{2}\left\|A u_{0}\right\|_{2}^{2}+\frac{\beta}{2}\left\|A^{1 / 2} u_{0}\right\|_{2}^{2 \gamma}\left\|A u_{0}\right\|_{2}^{2}+\frac{\epsilon}{2}\left\|A^{3 / 2} u_{0}\right\|_{2}^{2} \\
+\beta \gamma \int_{0}^{t}\|A u(s)\|_{2}^{2}\left\|A^{1 / 2} u(s)\right\|_{2}^{2(\gamma-1)}\left(A^{1 / 2} u^{\prime}(s), A^{1 / 2} u(s)\right) d s .
\end{aligned}
$$

In the case $N /(N-2) \leq q \leq \min \left\{(N+2) /(N-2),(N-2) /[N-4]^{+}\right\}$where $N \geq 3$, we have

$$
\begin{aligned}
& \left|\mu \int_{0}^{t}\left(A^{1 / 2}\left[|u(s)|^{q-1} u(s)\right], A^{1 / 2} u^{\prime}(s)\right) d s\right| \\
& \quad \leq q \mu \int_{0}^{t}\left\||u(s)|^{q-1} A^{1 / 2} u(s)\right\|_{2}\left\|A^{1 / 2} u^{\prime}(s)\right\|_{2} d s \\
& \quad \leq q \mu C \int_{0}^{t}\|u(s)\|_{(q-1) N}^{q-1}\left\|A^{1 / 2} u(s)\right\|_{2 N /(N-2)}\left\|A^{1 / 2} u^{\prime}(s)\right\|_{2} d s \\
& \quad \leq q \mu C \int_{0}^{t}\|u(s)\|_{(q-1) N}^{q-1}\|A u(s)\|_{2}\left\|A^{1 / 2} u^{\prime}(s)\right\|_{2} d s,
\end{aligned}
$$

where we have used Hölder's inequality and Sobolev-Poincaré's inequality. We observe from Gagliardo-Nirenberg inequality, Sobolev-Pointcaré's inequality, and (3.5) that

$$
\begin{aligned}
\|u(s)\|_{(q-1) N}^{q-1} & \leq C\|u(s)\|_{2 N /(N-2)}^{(q-1)(1-\theta)}\|A u(s)\|_{2}^{(q-1) \theta} \\
& \leq C\left\|A^{1 / 2} u(s)\right\|_{2}^{(q-1)(1-\theta)}\|A u(s)\|_{2}^{(q-1) \theta} \\
& \leq C M_{0}^{(q-1)(1-\theta) / 2}\|A u(s)\|_{2}^{(q-1) \theta} \text { with } \theta=\frac{N-2}{2}-\frac{1}{q-1}(<1) .
\end{aligned}
$$

Thus, (3.11) and (3.12) imply that

$$
\begin{aligned}
& \left|\mu \int_{0}^{t}\left(A^{1 / 2}\left[|u(s)|^{q-1} u(s)\right], A^{1 / 2} u^{\prime}(s)\right) d s\right| \\
& \quad \leq q \mu C M_{0}^{(q-1)(1-\theta) / 2} \int_{0}^{t}\|A u(s)\|_{2}^{1+(q-1) \theta}\left\|A^{1 / 2} u^{\prime}(s)\right\|_{2} d s .
\end{aligned}
$$

We also note that (3.5) implies

$$
\begin{aligned}
\beta \gamma \int_{0}^{t}\|A u(s)\|^{2} & \left\|A^{1 / 2} u(s)\right\|_{2}^{2(\gamma-1)}\left(A^{1 / 2} u^{\prime}(s), A^{1 / 2} u(s)\right) d s \\
& \leq \beta \gamma \int_{0}^{t}\|A u(s)\|_{2}^{2}\left\|A^{1 / 2} u^{\prime}(s)\right\|_{2}\left\|A^{1 / 2} u(s)\right\|_{2}^{2 \gamma-1} d s \\
& \leq \beta \gamma M_{0}^{(2 \gamma-1) / 2} \int_{0}^{t}\|A u(s)\|_{2}^{2}\left\|A^{1 / 2} u^{\prime}(s)\right\|_{2} d s .
\end{aligned}
$$


Consequently, (3.10), (3.13), and (3.14) give

$$
\begin{aligned}
\frac{1}{2}\left\|A^{1 / 2} u^{\prime}(t)\right\|_{2}^{2}+\frac{\alpha}{2}\|A u(t)\|_{2}^{2}+\frac{\epsilon}{2}\left\|A^{3 / 2} u(t)\right\|_{2}^{2} \\
+\frac{\beta}{2}\|A u(t)\|_{2}^{2}\left\|A^{1 / 2} u(t)\right\|_{2}^{2 \gamma}+\lambda \int_{0}^{t}\left\|A u^{\prime}(s)\right\|_{2}^{2} d s \\
\leq \frac{1}{2}\left\|A^{1 / 2} u_{1}\right\|_{2}^{2}+\frac{\alpha}{2}\left\|A u_{0}\right\|_{2}^{2}+\frac{\epsilon}{2}\left\|A^{3 / 2} u_{0}\right\|_{2}^{2} \\
+\frac{\beta}{2}\left\|A u_{0}\right\|_{2}^{2}\left\|A^{1 / 2} u_{0}\right\|_{2}^{2 \gamma}+q \mu C M_{0}^{(q-1)(1-\theta) / 2} \\
\quad \times \int_{0}^{t}\|A u(s)\|_{2}^{1+(q-1) \theta}\left\|A^{1 / 2} u^{\prime}(s)\right\|_{2} d s+\beta \gamma M_{0}^{(2 \gamma-1) / 2} \\
\quad \times \int_{0}^{t}\|A u(s)\|_{2}^{2}\left\|A^{1 / 2} u^{\prime}(s)\right\|_{2} d s .
\end{aligned}
$$

Set

$$
\begin{aligned}
E_{1}(t)= & \frac{1}{2}\left\|A^{1 / 2} u^{\prime}(t)\right\|_{2}^{2}+\frac{\alpha}{2}\|A u(t)\|_{2}^{2}+\frac{\epsilon}{2}\left\|A^{3 / 2} u(t)\right\|_{2}^{2} \\
& +\frac{\beta}{2}\|A u(t)\|_{2}^{2}\left\|A^{1 / 2} u(t)\right\|_{2}^{2 \gamma} .
\end{aligned}
$$

Then

$$
E_{1}(t) \leq E_{1}(0)+C_{1} \int_{0}^{t}\left(E_{1}(s)^{1+(q-1) \theta}+E_{1}(s)+E_{1}(s)^{2}\right) d s .
$$

Here, we set $g(s)=s+s^{1+(q-1) \theta}+s^{2}$ on $s \geq 0$. Then we have

$$
E_{1}(t) \leq E_{1}(0)+C_{1} \int_{0}^{t} g\left(E_{1}(s)\right) d s .
$$

Note that $g(s)$ is continuous and nondecreasing on $s \geq 0$. By applying BihariLangenhop's inequality (see [1]), we obtain

$$
E_{1}(t) \leq M_{1} \text { for some constant } M_{1}>0
$$

and so the desired result (3.8).

Proposition 3.5. If $\left(u_{0}, u_{1}\right)^{T} \in D\left(A^{4}\right) \times D\left(A^{2}\right)$ and

$$
\frac{N}{N-2} \leq q \leq \min \left\{\frac{N+2}{N-2}, \frac{N-2}{[N-4]^{+}}\right\} \quad(N \geq 3),
$$

then there exists a positive constant $M_{2}$, which does not depend on $\epsilon$, so that

$$
\sup _{t>0}\left\{\left\|A u^{\prime}(t)\right\|_{2}^{2},\left\|A^{3 / 2} u(t)\right\|_{2}^{2}, \epsilon\left\|A^{2} u(t)\right\|_{2}^{2}\right\} \leq M_{2} .
$$

Proof. Multiplying equation (2.5) by $A^{2} u^{\prime}(t)$,

$$
\begin{aligned}
& \frac{1}{2} \frac{d}{d t}\left(\left\|A u^{\prime}(t)\right\|_{2}^{2}+\alpha\left\|A^{3 / 2} u(t)\right\|_{2}^{2}+\epsilon\left\|A^{2} u(t)\right\|_{2}^{2}\right)+\lambda\left\|A^{3 / 2} u^{\prime}(t)\right\|_{2}^{2} \\
& \quad+\frac{\beta}{2}\left\|A^{1 / 2} u(t)\right\|_{2}^{2 \gamma} \frac{d}{d t}\left\|A^{3 / 2} u(t)\right\|_{2}^{2}+\mu\left(A^{1 / 2}\left[|u(t)|^{q-1} u(t)\right], A^{3 / 2} u^{\prime}(t)\right)=0 .
\end{aligned}
$$


Integrating (3.22) from 0 to $t$, we get

$$
\begin{aligned}
\frac{1}{2}\left(\left\|A u^{\prime}(t)\right\|_{2}^{2}+\alpha\left\|A^{3 / 2} u(t)\right\|_{2}^{2}+\epsilon\left\|A^{2} u(t)\right\|_{2}^{2}\right)+\frac{\beta}{2}\left\|A^{1 / 2} u(t)\right\|_{2}^{2 \gamma} \\
\quad \times\left\|A^{3 / 2} u(t)\right\|_{2}^{2}+\lambda \int_{0}^{t}\left\|A^{3 / 2} u^{\prime}(s)\right\|_{2}^{2} d s \\
\leq \frac{1}{2}\left(\left\|A u_{1}\right\|_{2}^{2}+\alpha\left\|A^{3 / 2} u_{0}\right\|_{2}^{2}+\epsilon\left\|A^{2} u_{0}\right\|_{2}^{2}\right)+\frac{\beta}{2}\left\|A^{1 / 2} u_{0}\right\|_{2}^{2 \gamma}\left\|A^{3 / 2} u_{0}\right\|_{2}^{2} \\
\quad+\mu \int_{0}^{t}\left(A^{1 / 2}\left[|u(s)|^{q-1} u(s)\right], A^{3 / 2} u^{\prime}(s)\right) d s \\
\quad+\beta \gamma \int_{0}^{t}\left\|A^{1 / 2} u(s)\right\|_{2}^{2(\gamma-1)}\left(A^{1 / 2} u^{\prime}(s), A^{1 / 2} u(s)\right)\left\|A^{3 / 2} u(s)\right\|_{2}^{2} d s .
\end{aligned}
$$

In the case $(N /(N-2)) \leq q \leq \min \left\{(N+2) /(N-2),(N-2) /[N-4]^{+}\right\}$where $(N \geq 3)$, we have

$$
\begin{aligned}
\mu\left(A^{1 / 2}\right. & {\left.\left[|u(s)|^{q-1} u(s)\right], A^{3 / 2} u^{\prime}(s)\right) } \\
& \leq q \mu\left\||u(s)|^{q-1} A^{1 / 2} u(s)\right\|_{2}\left\|A^{3 / 2} u^{\prime}(s)\right\|_{2} \\
& \leq q \mu C\|u(s)\|_{(q-1) N}^{q-1}\left\|A^{1 / 2} u(s)\right\|_{2 N /(N-2)}\left\|A^{3 / 2} u^{\prime}(s)\right\|_{2} \\
& \leq q \mu C\left\|A^{1 / 2} u(s)\right\|_{2}^{q-1}\|A u(s)\|_{2}\left\|A^{3 / 2} u^{\prime}(s)\right\|_{2} .
\end{aligned}
$$

Thus (3.5), (3.8), and (3.24) imply that

$$
\begin{aligned}
\mu\left(A^{1 / 2}\left[|u(s)|^{q-1} u(s)\right], A^{3 / 2} u^{\prime}(s)\right) & \leq q \mu C M_{0}^{(q-1) / 2} M_{1}^{1 / 2}\left\|A^{3 / 2} u^{\prime}(s)\right\|_{2} \\
& \leq \frac{1}{2 \lambda}(q \mu C)^{2} M_{0}^{q-1} M_{1}+\frac{\lambda}{2}\left\|A^{3 / 2} u^{\prime}(s)\right\|_{2}^{2}
\end{aligned}
$$

and so

$$
\begin{aligned}
\mu \int_{0}^{t}\left(A^{1 / 2}[\right. & \left.\left.|u(s)|^{q-1} u(s)\right], A^{3 / 2} u^{\prime}(s)\right) d s \\
& \leq \frac{1}{2 \lambda}(q \mu C)^{2} M_{0}^{q-1} M_{1} T+\frac{\lambda}{2} \int_{0}^{t}\left\|A^{3 / 2} u^{\prime}(s)\right\|_{2}^{2} d s .
\end{aligned}
$$

On the other hand, (3.5) and (3.8) imply that

$$
\begin{array}{rl}
\beta \gamma \int_{0}^{t} \| A^{1 / 2} & u(s)\left\|_{2}^{2(\gamma-1)}\left(A^{1 / 2} u^{\prime}(s), A^{1 / 2} u(s)\right)\right\| A^{3 / 2} u(s) \|_{2}^{2} d s \\
\leq & \beta \gamma \int_{0}^{t}\left\|A^{1 / 2} u(s)\right\|_{2}^{2 \gamma-1}\left\|A^{1 / 2} u^{\prime}(s)\right\|_{2}\left\|A^{3 / 2} u(s)\right\|_{2}^{2} d s \\
\leq & \beta \gamma M_{0}^{(2 \gamma-1) / 2} M_{1}^{1 / 2} \int_{0}^{t}\left\|A^{3 / 2} u(s)\right\|_{2}^{2} d s .
\end{array}
$$

Thus, from (3.23), (3.26), and (3.27),

$$
\begin{aligned}
\frac{1}{2}\left(\left\|A u^{\prime}(t)\right\|_{2}^{2}\right. & \left.+\alpha\left\|A^{3 / 2} u(t)\right\|_{2}^{2}+\epsilon\left\|A^{2} u(t)\right\|_{2}^{2}+\beta\left\|A^{1 / 2} u(t)\right\|_{2}^{2 \gamma}\left\|A^{3 / 2} u(t)\right\|_{2}^{2}\right) \\
& +\frac{\lambda}{2} \int_{0}^{t}\left\|A^{3 / 2} u^{\prime}(s)\right\|_{2}^{2} d s \\
\leq & \frac{1}{2}\left(\left\|A u_{1}\right\|_{2}^{2}+\alpha\left\|A^{3 / 2} u_{0}\right\|_{2}^{2}+\epsilon\left\|A^{2} u_{0}\right\|_{2}^{2}\right)+\frac{\beta}{2}\left\|A^{1 / 2} u_{0}\right\|^{2 \gamma}\left\|A^{3 / 2} u_{0}\right\|^{2} \\
& +\frac{1}{2 \lambda}(q \mu C)^{2} M_{0}^{q-1} M_{1} T+\beta \gamma M_{0}^{(2 \gamma-1) / 2} M_{1}^{1 / 2} \int_{0}^{t}\left\|A^{3 / 2} u(s)\right\|_{2}^{2} d s .
\end{aligned}
$$


Thus,

$$
\begin{aligned}
E_{2}(t)+\frac{\lambda}{2} \int_{0}^{t}\left\|A^{3 / 2} u^{\prime}(s)\right\|_{2}^{2} d s & \leq E_{2}(0)+C_{1}+C_{2} \int_{0}^{t} \alpha\left\|A^{3 / 2} u(s)\right\|_{2}^{2} d s \\
& \leq E_{2}(0)+C_{1}+C_{2} \int_{0}^{t} E_{2}(s) d s,
\end{aligned}
$$

where

$$
\begin{gathered}
E_{2}(t)=\frac{1}{2}\left(\left\|A u^{\prime}(t)\right\|_{2}^{2}+\alpha\left\|A^{3 / 2} u(t)\right\|_{2}^{2}+\epsilon\left\|A^{2} u(t)\right\|_{2}^{2}+\beta\left\|A^{1 / 2} u(t)\right\|_{2}^{2 \gamma}\left\|A^{3 / 2} u(t)\right\|_{2}^{2}\right), \\
C_{1}=\frac{1}{2 \lambda}(q \mu C)^{2} M_{0}^{q-1} M_{1} T, \quad C_{2}=\frac{1}{\alpha} \beta \gamma M_{0}^{(2 \gamma-1) / 2} M_{1}^{1 / 2} .
\end{gathered}
$$

Applying Gronwall's inequality, we easily obtain the desired result.

Proposition 3.6. If $T>0$ and $\left(u_{0}, u_{1}\right)^{T} \in D\left(A^{2}\right) \times D(A)$ and

$$
\frac{N}{N-2} \leq q \leq \min \left\{\frac{N+2}{N-2}, \frac{N-2}{[N-4]^{+}}\right\} \quad(N \geq 3),
$$

then there exists a positive constant $N$, which does not depend on $\epsilon$, so that

$$
\sup _{t>0}\left\{\left\|u^{\prime \prime}(t)\right\|_{2}^{2}\right\} \leq N \text {. }
$$

Proof. Multiplying the differentiated equation of (2.5) in $t$ by $u^{\prime \prime}(t)$, we get

$$
\begin{aligned}
\frac{1}{2} \frac{d}{d t} \| & u^{\prime \prime}(t)\left\|_{2}^{2}+\frac{\alpha}{2} \frac{d}{d t}\right\| A^{1 / 2} u^{\prime}(t)\left\|_{2}^{2}+\frac{\epsilon}{2} \frac{d}{d t}\right\| A u^{\prime}(t)\left\|_{2}^{2}+\lambda\right\| A^{1 / 2} u^{\prime \prime}(t) \|_{2}^{2} \\
+ & \frac{\beta}{2}\left\|A^{1 / 2} u(t)\right\|_{2}^{2 \gamma}\left(A u^{\prime}(t), u^{\prime \prime}(t)\right)+q \mu \int_{\Omega}|u(t)|^{q-1} u^{\prime}(t) u^{\prime \prime}(t) d x \\
+ & 2 \beta \gamma\left\|A^{1 / 2} u(t)\right\|_{2}^{2(\gamma-1)}\left(A^{1 / 2} u(t), A^{1 / 2} u^{\prime}(t)\right)\left(A u(t), u^{\prime \prime}(t)\right)=0 .
\end{aligned}
$$

Note that if $(N /(N-2)) \leq q \leq \min \left\{(N+2) /(N-2),(N-2) /[N-4]^{+}\right\}$where $N \geq 3$, then (3.5) and (3.8) imply that

$$
\begin{aligned}
q \mu \mid \int_{\Omega} & |u(t)|^{q-1} u^{\prime}(t) u^{\prime \prime}(t) d x \mid \\
& \leq q \mu\|u(t)\|_{(q-1) N}^{q-1}\left\|u^{\prime}(t)\right\|_{2 N /(N-2)}\left\|u^{\prime \prime}(t)\right\|_{2} \\
& \leq q \mu C\|u(t)\|_{2 N /(N-2)}^{(q-1)(1-\theta)}\|A u(t)\|^{(q-1) \theta}\left\|u^{\prime}(t)\right\|_{2 N /(N-2)}\left\|u^{\prime \prime}(t)\right\|_{2} \\
& \leq q \mu C\left\|A^{1 / 2} u(t)\right\|_{2}^{(q-1)(1-\theta)}\|A u(t)\|^{(q-1) \theta}\left\|A^{1 / 2} u^{\prime}(t)\right\|_{2}\left\|u^{\prime \prime}(t)\right\|_{2} \\
& \leq q \mu C M_{0}^{((q-1)(1-\theta)) / 2} M_{1}^{(1+(q-1) \theta) / 2}\left\|u^{\prime \prime}(t)\right\|_{2} .
\end{aligned}
$$

Also, (3.5) and (3.8) give

$$
\begin{aligned}
2 \beta \gamma \| A^{1 / 2} u(t) & \|_{2}^{2(\gamma-1)}\left|\left(A^{1 / 2} u(t), A^{1 / 2} u^{\prime}(t)\right)\left(A u(t), u^{\prime \prime}(t)\right)\right| \\
& \leq 2 \beta \gamma\left\|A^{1 / 2} u(t)\right\|_{2}^{2 \gamma-1}\left\|A^{1 / 2} u^{\prime}(t)\right\|_{2}\|A u(t)\|_{2}\left\|u^{\prime \prime}(t)\right\|_{2} \\
& \leq 2 \beta \gamma M_{0}^{(2 \gamma-1) / 2} M_{1}\left\|u^{\prime \prime}(t)\right\|_{2}
\end{aligned}
$$


and

$$
\begin{aligned}
\frac{\beta}{2}\left\|A^{1 / 2} u(t)\right\|_{2}^{2 \gamma}\left(A u^{\prime}(t), u^{\prime \prime}(t)\right) & \leq \frac{\beta}{2}\left\|A^{1 / 2} u(t)\right\|_{2}^{2 \gamma}\left\|A u^{\prime}(t)\right\|_{2}\left\|u^{\prime \prime}(t)\right\|_{2} \\
& \leq \frac{\beta}{2} M_{0}^{\gamma}\left\|A u^{\prime}(t)\right\|_{2}\left\|u^{\prime \prime}(t)\right\|_{2} .
\end{aligned}
$$

Thus, from (3.33), (3.34), (3.35), and (3.36), we get

$$
\begin{aligned}
\frac{1}{2} \frac{d}{d t}\left\|u^{\prime \prime}(t)\right\|_{2}^{2}+\frac{\alpha}{2} \frac{d}{d t}\left\|A^{1 / 2} u^{\prime}(t)\right\|_{2}^{2}+\frac{\epsilon}{2} \frac{d}{d t}\left\|A u^{\prime}(t)\right\|_{2}^{2}+\lambda\left\|A^{1 / 2} u^{\prime \prime}(t)\right\|_{2}^{2} \\
\leq\left(q \mu C M_{0}^{((q-1)(1-\theta)) / 2} M_{1}^{(1+(q-1) \theta) / 2}+2 \beta \gamma M_{0}^{(2 \gamma-1) / 2} M_{1}\right)\left\|u^{\prime \prime}(t)\right\|_{2} \\
+\frac{\beta}{2} M_{0}^{\gamma}\left\|A u^{\prime}(t)\right\|_{2}\left\|u^{\prime \prime}(t)\right\|_{2} .
\end{aligned}
$$

Integrating (3.37) from 0 to $t$, we get

$$
\begin{aligned}
E_{3}(t)+\lambda \int_{0}^{t}\left\|A^{1 / 2} u^{\prime \prime}(s)\right\|_{2}^{2} d s & \leq E_{3}(0)+C_{3} \int_{0}^{t}\left(\left\|A u^{\prime}(s)\right\|_{2}\left\|u^{\prime \prime}(s)\right\|_{2}+\left\|u^{\prime \prime}(s)\right\|_{2}\right) d s \\
& \leq E_{3}(0)+C_{4} \int_{0}^{t}\left(E_{3}(s)^{1 / 2}+E_{3}(s)\right) d s,
\end{aligned}
$$

where

$$
\begin{gathered}
E_{3}(t)=\frac{1}{2}\left\|u^{\prime \prime}(t)\right\|_{2}^{2}+\frac{\alpha}{2}\left\|A^{1 / 2} u^{\prime}(t)\right\|_{2}^{2}+\frac{\epsilon}{2}\left\|A u^{\prime}(t)\right\|_{2}^{2}, \\
C_{3}=\max \left\{q \mu C M_{0}^{((q-1)(1-\theta)) / 2} M_{1}^{(1+(q-1) \theta) / 2}, 2 \beta \gamma M_{0}^{(2 \gamma-1) / 2} M_{1}, \frac{\beta}{2} M_{0}^{\gamma}\right\} .
\end{gathered}
$$

Here, we set $g(s)=s^{1 / 2}+s$ on $s \geq 0$. Then we have

$$
E_{3}(t) \leq E_{3}(0)+C_{4} \int_{0}^{t} g\left(E_{3}(s)\right) d s .
$$

Note that $g(s)$ is continuous and nondecreasing on $s \geq 0$. By applying BihariLangenhop's inequality (see [1]), we obtain

$$
E_{3}(t) \leq M_{2} \text { for some constant } M_{2}>0
$$

and so we have the desired result (3.32).

4. Convergence results. In this section, we establish the uniform convergence of strong solutions to (2.5) as $\epsilon \rightarrow 0$. At this point, we find it advantageous to make the dependence of solutions to (2.5) on $\epsilon$ explicit. To be more precise, we let $U_{\epsilon}(t)=$ $\left(u_{\epsilon}(t), u_{\epsilon}^{\prime}(t)\right)$ be the solution to (2.12) and observe that $U_{\epsilon}$ is the solution of (2.5). Continuing in this manner, $\left(u_{\epsilon}(t)\right)(x)=u_{\epsilon}(x, t)$ satisfies (2.5). We are concerned with the convergence of solutions to (1.1) on finite interval of arbitrary length. In what follows, we let $T>0$ and consider the convergence on $[0, T]$. In particular, we want to establish that

$$
\lim _{\epsilon \rightarrow 0+}\left(\sup _{t \in[0, T]}\left\|u_{\epsilon}(\cdot, t)-u(\cdot, t)\right\|_{\infty}\right)=0,
$$


where $u$ is the strong solution to (1.1). Our proofs rely upon the classical Arzela-Ascoli arguments and the uniqueness of solutions to (1.1).

LEMMA 4.1. Let $\left\{\epsilon_{n}\right\}$ converge to zero and $\left(u_{0}, u_{1}\right)^{T}=U_{0} \in D\left(A^{2}\right) \times D\left(A^{2}\right)$. If, for each $\epsilon_{n}, u_{\epsilon_{n}}$ is a solution to (2.5), then there exists a subsequence $\epsilon_{n^{\prime}} \rightarrow 0$ and $u_{*} ;[0, T] \rightarrow H_{A^{1 / 2}}$ such that

$$
\lim _{n \rightarrow \infty}\left\|u_{\epsilon_{n^{\prime}}}(t)-u_{*}(t)\right\|_{A^{1 / 2}}=0 \quad \text { uniformly for } t \in[0, T] .
$$

Moreover, if $\left(u_{\epsilon_{n^{\prime}}}(t)\right)(x)=u_{\epsilon_{n^{\prime}}}(x, t)$ and $\left(u_{*}(t)\right)(x)=u_{*}(x, t)$, then

$$
\lim _{n \rightarrow \infty}\left\|u_{\epsilon_{n^{\prime}}}(\cdot, t)-u_{*}(\cdot, t)\right\|_{\infty}=0 \quad \text { uniformly for } t \in[0, T] .
$$

Proof. From Proposition 3.4, we observe that

$$
\begin{aligned}
\left\|A^{1 / 2} u_{\epsilon_{n}}(t)-A^{1 / 2} u_{\epsilon_{n}}(s)\right\|_{2} & \leq \int_{s}^{t}\left\|A^{1 / 2} u_{\epsilon_{n}}^{\prime}(r)\right\|_{2} d r \\
& \leq M_{1}^{1 / 2}|t-s| \text { for } t, s \in[0, T] .
\end{aligned}
$$

Moreover, we also have from Proposition 3.3

$$
\left\|A^{1 / 2} u_{\epsilon_{n}}(t)\right\|_{2} \leq M_{0}^{1 / 2} \text { for } t \in[0, T] .
$$

Thus, the above results, together with the compactness of $A^{-1 / 2}$, imply that the sequence $\left\{u_{\epsilon_{n}}(t)\right\}$ is uniformly bounded and uniformly equicontinuous in $H_{A^{1 / 2}}$. Hence, we can apply Arzela-Ascoli theorem to the sequence $\left\{u_{\epsilon_{n}}(t)\right\}$ in $H_{A^{1 / 2}}$. Thus, we can find a subsequence $\left\{u_{\epsilon_{n^{\prime}}}\right\}$ and the limit function $u_{*}(t) ;[0, T] \rightarrow H_{A^{1 / 2}}$ such that

$$
u_{\epsilon_{n}}(t) \longrightarrow u_{*}(t) \text { in } H_{A^{1 / 2}} \text { uniformly for } t \in[0, T] .
$$

The final assertion follows immediately from the Sobolev embedding theorem.

Now, subsequent results presuppose that the hypotheses of Lemma 4.1 remain in effect.

Proposition 4.2. If we define $f_{\epsilon_{n}}:[0, T] \rightarrow H$ by

$$
\begin{aligned}
f_{\epsilon_{n}}(t) & =\pi_{2}\left(F_{\epsilon_{n}}\left(u_{\epsilon_{n}}(t)\right)\right) \\
& =-\beta\left\|A^{1 / 2} u_{\epsilon_{n}}(t)\right\|_{2}^{2 \gamma} A u_{\epsilon_{n}}(t)-\epsilon_{n} A^{2} u_{\epsilon_{n}}(t)-\mu\left|u_{\epsilon_{n}}(t)\right|^{q-1} u_{\epsilon_{n}}(t),
\end{aligned}
$$

then $\left\{f_{\epsilon_{n}}(t)\right\}$ converges weakly to $f_{*}(t)$ in $H$ on $[0, T]$, where $f_{*}(t)$ is defined by

$$
f_{*}(t)=-\beta\left\|A^{1 / 2} u_{*}(t)\right\|_{2}^{2 \gamma} A u_{*}(t)-\mu\left|u_{*}(t)\right|^{q-1} u_{*}(t)
$$

Proof. Since $\lim _{n \rightarrow \infty} u_{\epsilon_{n}}(t)=u_{*}(t)$ in $H_{A^{1 / 2}}$, we have

$$
\lim _{n \rightarrow \infty}\left\|A^{1 / 2} u_{\epsilon_{n}}(t)\right\|_{2}^{2 \gamma}=\lim _{n \rightarrow \infty}\left\|u_{\epsilon_{n}}(t)\right\|_{A^{1 / 2}}^{2 \gamma}=\left\|u_{*}(t)\right\|_{A^{1 / 2}}^{2 \gamma}=\left\|A^{1 / 2} u_{*}(t)\right\|_{2}^{2 \gamma} .
$$

By virtue of Proposition 3.4, the sequence $\left\{A u_{\epsilon_{n}}(t)\right\}$ is uniformly bounded in $H$ for $t \in[0, T]$. Moreover, $\left\{u_{\epsilon_{n}}(t)\right\}$ converges to $u_{*}(t)$ in $H$. Since $A$ is closed, we have $u_{*}(t) \in D(A)$ and $A u_{\epsilon_{n}}(t)$ converges weakly to $A u_{*}(t)$ in $H$. Note that $\left\{\epsilon_{n} u_{\epsilon_{n}}(t)\right\}$ converges to zero as $n \rightarrow \infty$ and $\left\{\epsilon_{n} A^{2} u_{\epsilon_{n}}(t)\right\}=\left\{A^{2}\left(\epsilon_{n} u_{\epsilon_{n}}(t)\right)\right\}$ is uniformly bounded in $H$. Since $A^{2}$ is closed, $\left\{\epsilon_{n} A^{2} u_{\epsilon_{n}}\right\}$ converges weakly to zero. This completes the proof. 
Proposition 4.3. The sequence $\left\{u_{\epsilon_{n}}(t)\right\}$ converges weakly in $X$. Moreover, $u_{*}^{\prime}(t)$ exists and $u_{\epsilon_{n}}^{\prime}(t)$ converges weakly to $u_{*}^{\prime}(t)$ for a.e. $t \in[0, T]$.

Proof. Since $X$ is a product of Hilbert spaces, it is of course reflexive. Thus, $\left\{U_{\epsilon_{n}}(t)\right\}=\left\{\left(u_{\epsilon_{n}}(t), u_{\epsilon_{n}}^{\prime}(t)\right)^{T}\right\}$ is a bounded sequence in a reflexive Banach space $X$ and so it must have a subsequence $\left\{U_{\epsilon_{n^{\prime}}}(t)\right\}=\left\{\left(u_{\epsilon_{n^{\prime}}}(t), u_{\epsilon_{n^{\prime}}}^{\prime}(t)\right)^{T}\right\}$ such that $\left\{U_{\epsilon_{n^{\prime}}}(t)\right\}$ converges weakly to $\left\{U_{*}(t)\right\}$.

But Lemma 4.1 implies that $\left\{U_{*}(t)\right\}=\left\{\left(u_{*}(t), u_{*}^{\prime}(t)\right)^{T}\right\}$.

Proposition 4.4. The function $F_{*}(t)$, defined by

$$
F_{*}(t)=\left(0, f_{*}(t)\right)^{T}=\left(0,-\beta\left\|A^{1 / 2} u_{*}(t)\right\|_{2}^{2 \gamma} A u_{*}(t)-\mu\left|u_{*}(t)\right|^{q-1} u_{*}(t)\right)^{T},
$$

is differentiable a.e. $t \in[0, T]$.

Proof. Note that $A^{1 / 2} u_{*}(\cdot)$ is the limit of a uniformly convergent sequence of uniformly Lipschitz continuous functions in reflexive Banach space $H$ and so it is differentiable almost everywhere. Thus, $\left\|A^{1 / 2} u_{*}(\cdot)\right\|_{2}^{2 \gamma}$ is differentiable for a.e. $t \in[0, T]$. And $d / d t\left(A u_{*}(t)\right)$ exists since it is a weak limit of a bounded sequence $d / d t\left(A u_{\epsilon_{n^{\prime}}}(t)\right)$.

LEMMA 4.5. Let $u(t)$ be the unique solution to (2.18) represented by (2.19) on $[0, T]$. If $u_{0}=\left(u_{0}, u_{1}\right)^{T} \in D\left(A^{4}\right) \times D\left(A^{2}\right)$ and $\epsilon_{n} \rightarrow 0$, then $u_{\epsilon_{n}}(t)$ converges weakly to $u(t)$ in $X$ on $[0, T]$.

Proof. Note that solutions to (2.5) have abstract variation of parameters representation

$$
U_{\epsilon_{n}}(t)=T(t) U_{0}+\int_{0}^{t} T(t-s) F\left(U_{\epsilon_{n}}(s)\right) d s=T(t) U_{0}+\int_{0}^{t} T(t-s) \bar{f}_{\epsilon_{n}}(s) d s,
$$

where

$$
\begin{aligned}
\bar{f}_{\epsilon_{n}}(t) & =\left(0, f_{\epsilon_{n}}(t)\right)^{T} \\
& =\left(0,-\beta\left\|A^{1 / 2} u_{\epsilon_{n}}(t)\right\|_{2}^{2 \gamma}-\epsilon_{n} A^{2} u_{\epsilon_{n}}(t)-\mu\left|u_{\epsilon_{n}}(t)\right|^{q-1} u_{\epsilon_{n}}(t)\right)^{T} .
\end{aligned}
$$

If $W \in X$ and $\langle\cdot, \cdot\rangle_{X}$ denotes the inner product of $X$, we apply $W$ to each side of (4.11) and take the inner product to obtain

$$
\left\langle U_{\epsilon_{n}}(t), W\right\rangle_{X}=\left\langle T(t) U_{0}, W\right\rangle_{X}+\int_{0}^{t}\left\langle T(t-s) \bar{f}_{\epsilon_{n}}(s), W\right\rangle_{X} d s .
$$

We have shown that $U_{\epsilon_{n}}(t)=\left(u_{\epsilon_{n}}(t), u_{\epsilon_{n}}^{\prime}(t)\right)^{T}$ has a weakly convergent subsequence which we denote by $U_{\epsilon_{m}}(t)=\left(u_{\epsilon_{m}}(t), u_{\epsilon_{m}}^{\prime}(t)\right)^{T}$. The limit of this subsequence, for the time being, is denoted by $U_{*}(t)=\left(u_{*}(t), u_{*}^{\prime}(t)\right)^{T}$. Additionally, we have shown that $\bar{f}_{\epsilon_{m}}(t)=\left(0, f_{\epsilon_{m}}(t)\right)^{T}$ converges weakly to $\bar{f}_{*}(t)=\left(0, f_{*}(t)\right)^{T}=\left(0,-\beta\left\|A^{1 / 2} u_{*}(t)\right\|_{2}^{2 \gamma}-\right.$ $\left.\mu\left|u_{*}(t)\right|^{q-1} u_{*}(t)\right)^{T}$. We may compute the limit as $m \rightarrow \infty$ of each side of (4.13) to produce

$$
\left\langle U_{*}(t), W\right\rangle_{X}=\left\langle T(t) U_{0}, W\right\rangle_{X}+\int_{0}^{t}\left\langle T(t-s) \bar{f}_{*}(s), W\right\rangle_{X} d s
$$


Standard techniques yield

$$
U_{*}(t)=T(t) U_{0}+\int_{0}^{t} T(t-s) \bar{f}_{*}(s) d s .
$$

The differentiability of $f_{*}(t)$, together with the regularity results for abstract Cauchy initial value problem (cf. Pazy [10, Chapter 4]), allows us to differentiate (4.15) to produce a strong solution to (2.18). However, solutions of (2.18) are unique and, therefore,

$$
U_{*}(t)=\left(u_{*}(t), u_{*}^{\prime}(t)\right)^{T}=U(t)=\left(u(t), u^{\prime}(t)\right)^{T} .
$$

Now, we are in a position to obtain our result.

THEOREM 4.6. If $\left(u_{0}, u_{1}\right)^{T}=U_{0} \in D\left(A^{4}\right) \times D\left(A^{2}\right)$ and $T>0$ and

$$
\frac{N}{N-2} \leq q \leq \min \left\{\frac{N+2}{N-2}, \frac{N-2}{[N-4]^{+}}\right\} \quad(N \geq 3),
$$

then

$$
\lim _{\epsilon \rightarrow 0+} \sup _{t \in[0, T]}\left\|u_{\epsilon}(t)-u(t)\right\|_{\infty}=0,
$$

where $u_{\epsilon}$ and $u$ are strong solutions to (1.1).

Proof. We pick an arbitrary sequence $\left\{\epsilon_{n}\right\}$ such that $\epsilon_{n} \rightarrow 0$. Lemma 4.1 along with Lemma 4.5 give our desired result.

ACKNOWLEDGEMENT. This work is supported by KOSEF 1996.

\section{REFERENCES}

[1] E. F. Beckenbach and R. Bellman, Inequalities, Springer-Verlag, New York, Inc., 1965. MR 33\#236. Zbl 206.06802.

[2] M. Da Silva Alves, Variational inequality for a nonlinear model of the oscillations of beams, Nonlinear Anal. 28 (1997), no. 6, 1101-1108. MR 97k:35253. Zbl 871.35064.

[3] W. E. Fitzgibbon and M. E. Parrott, Convergence of singular perturbations of strongly damped nonlinear wave equations, Nonlinear Anal. 28 (1997), no. 1, 165-174. MR 97j:35095. Zbl 866.35016.

[4] M. P. Matos and D. C. Pereira, On a hyperbolic equation with strong damping, Funkcial. Ekvac. 34 (1991), no. 2, 303-311. MR 92j:34117. Zbl 746.34039.

[5] T. Matsuyama and R. Ikehata, On global solutions and energy decay for the wave equations of Kirchhoff type with nonlinear damping terms, J. Math. Anal. Appl. 204 (1996), no. 3, 729-753. MR 98f:35109. Zbl 970.28156.

[6] K. Narasimha, Nonlinear vibration of an elastic string, J. Sound Vibration 8 (1968), 134146.

[7] K. Nishihara and Y. Yamada, On global solutions of some degenerate quasilinear hyperbolic equations with dissipative terms, Funkcial. Ekvac. 33 (1990), no. 1, 151-159. MR 91f:35181. Zbl 715.35053.

[8] K. Ono, Global existence, decay, and blowup of solutions for some mildly degenerate nonlinear Kirchhoff strings, J. Differential Equations 137 (1997), no. 2, 273-301. MR 98f:35149. Zbl 879.35110.

[9] K. Ono and K. Nishihara, On a nonlinear degenerate integro-differential equation of hyperbolic type with a strong dissipation, Adv. Math. Sci. Appl. 5 (1995), no. 2, 457-476. MR 97g:35115. Zbl 842.45005. 
[10] A. Pazy, Semigroups of Linear Operators and Applications to Partial Differential Equations, vol. 44, Springer-Verlag, New York, 1983. MR 85g:47061. Zbl 516.47023.

[11] S. I. Pohozaev, On a class of quasilinear hyperbolic equations, Math. USSR-Sb. 25 (1975), 145-158. Zbl 328.35060.

[12] G. F. Webb, Existence and asymptotic behavior for a strongly damped nonlinear wave equation, Canad. J. Math. 32 (1980), no. 3, 631-643. MR 81i:35116. Zbl 432.35046.

PARK ANd Bae: Department of Mathematics, Pusan National UniVersity, Pusan 609735, KOREA 


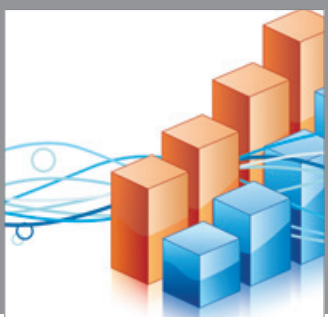

Advances in

Operations Research

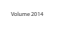

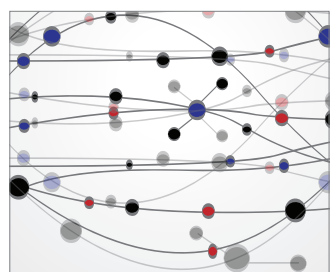

\section{The Scientific} World Journal
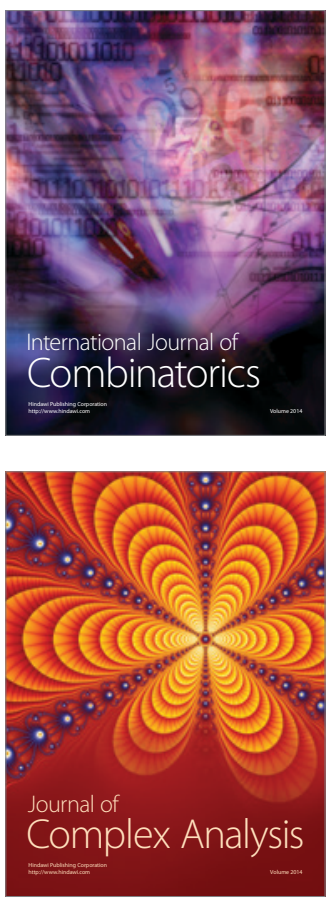

International Journal of

Mathematics and

Mathematical

Sciences
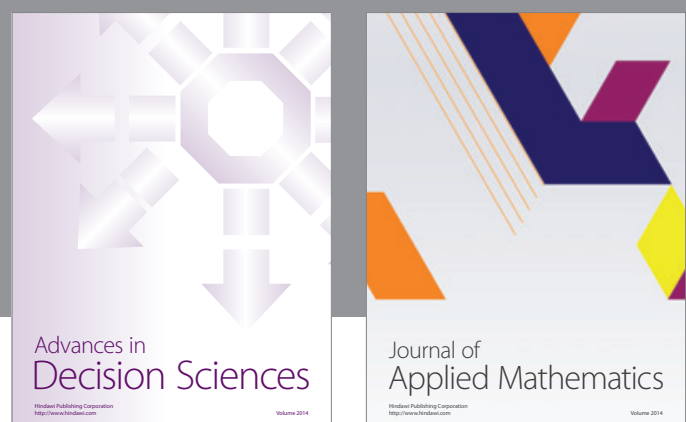

Journal of

Applied Mathematics
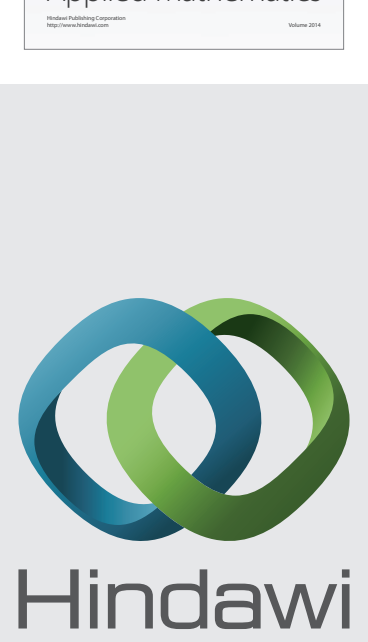

Submit your manuscripts at http://www.hindawi.com
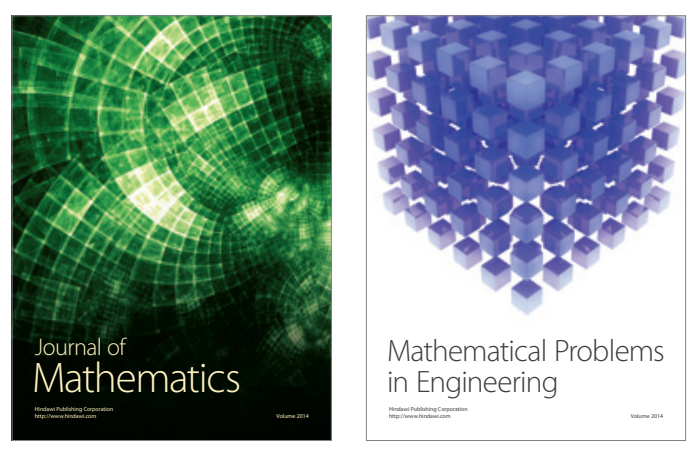

Mathematical Problems in Engineering
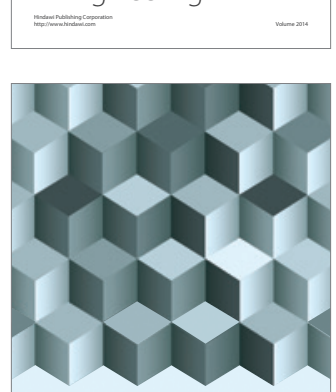

Journal of

Function Spaces
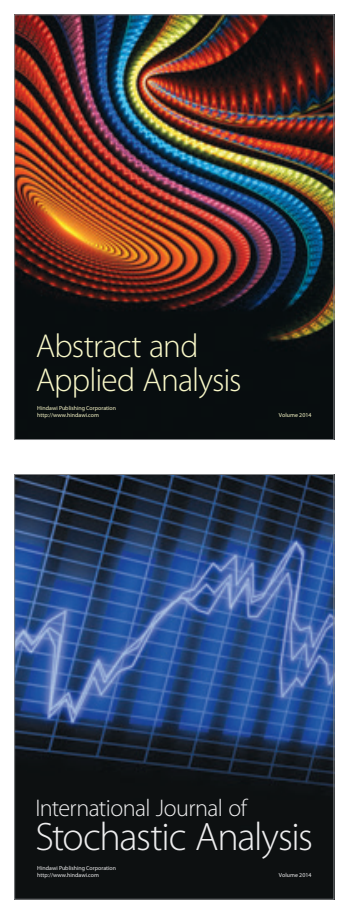

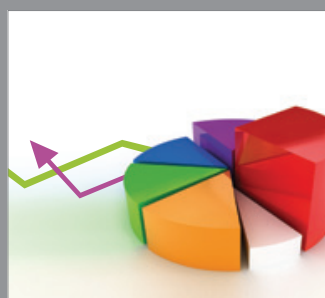

ournal of

Probability and Statistics

Promensencen
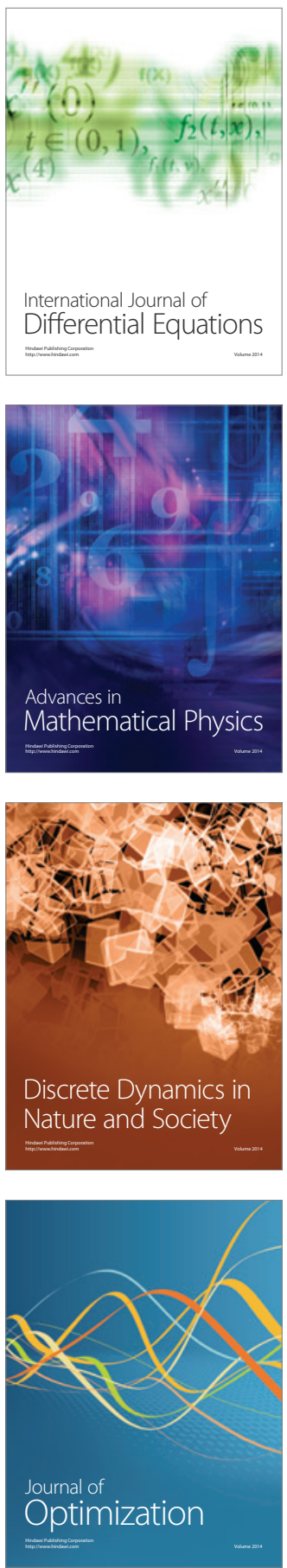